\title{
Dynamiques de décomposition des résidus de cultures sur des exploitations pratiquant l'agriculture de conservation en région Grand Est, France
}

\author{
Pascal Thiebeau* et Sylvie Recous \\ INRA, UMR 614 Fractionnement des Agro-Ressources et Environnement (FARE), 2, Esplanade R. Garros, 51100 Reims, France
}

\begin{abstract}
Résumé - La réduction du travail du sol, dans les exploitations agricoles pratiquant l'agriculture de conservation, conduit à laisser les résidus de culture à la surface des sols après la récolte, mais peu de références sont disponibles sur leur décomposition. L'objectif du travail était de déterminer la dynamique de décomposition des résidus de cultures dans ces situations en systèmes de travail réduit du sol et en semis direct, pour, à terme, mieux les gérer et tenir compte de leur contribution à la fertilisation des cultures suivantes. L'étude a consisté à suivre, de 2009 à 2011, sur quatre exploitations agricoles de la région Grand Est (France), la décomposition de résidus de cultures de maïs grain, tournesol, colza, blé et escourgeon, et le carbone $(\mathrm{C})$ et l'azote $(\mathrm{N})$ de ces résidus. Le suivi a été fait par le prélèvement et l'analyse des biomasses de résidus pendant une à deux années après les récoltes. L'expression du temps, en utilisant la méthode des jours normalisés à $15^{\circ} \mathrm{C}$, a permis de comparer les cinétiques des différents sites et des trois années. La décomposition a été rapide dans toutes les situations, la quasi-totalité des résidus étant dégradée après 700 jours normalisés à $15^{\circ} \mathrm{C}$. Les cinétiques d'évolution des quantités de biomasse, $\mathrm{C}$ et $\mathrm{N}$ ont suivi une relation mathématique unique en fonction du temps normalisé, quelles que soient l'espèce, la localisation et l'année. Ces cinétiques peuvent donc être prédites par un seul et même ajustement non linéaire, statistiquement très hautement significatif. Ceci indique que, dans les conditions agricoles et climatiques explorées de la région Grand Est, la température est le principal facteur déterminant la cinétique de décomposition des résidus de culture, rendant relativement simple une prise en compte de la minéralisation de l'azote des résidus de culture dans le calcul des bilans d'azote pour les cultures suivantes.
\end{abstract}

Mots clés : travail du sol réduit / dynamiques de décomposition de résidus de cultures / carbone / azote / modélisation

\begin{abstract}
Crop residues decomposition dynamics in farms practising conservation agriculture in the Grand Est region, France. Reduced tillage of farms practicing conservation agriculture leads to leaving crop residues on the soil surface after harvesting, but few references are available on their in situ field decomposition. This study aimed to determine residues decomposition through their dry matter, carbon (C) and nitrogen $(\mathrm{N})$ dynamics in reduced tillage and direct sowing cropping systems, to improve their management and $\mathrm{N}$ contribution over time to the following crops. Maize, sunflower, rapeseed, winter wheat and winter barley residues decomposition were recorded in four farms in the Grand Est region (France) from 2009 to 2011. Monitoring was done by sampling and analysis of residual biomass, during one to two years after harvest. The expression of time, based on normalized days at $15^{\circ} \mathrm{C}$, permitted to compare crop residue dynamics for the different sites and the three years. Decomposition was rapid in all situations, with almost all residues disappearing after 700 normalized days at $15^{\circ} \mathrm{C}$. The kinetics of biomass, $\mathrm{C}$ and $\mathrm{N}$ followed a common mathematical relationship as a function of normalized time, regardless of species, location and year. These kinetics can be predicted by a single nonlinear equation, statistically very highly significant. This indicates that, under the explored agricultural and climatic conditions of the Grand Est region, temperature was the main factor determining the kinetics of crop residue decomposition, making it relatively simple to estimate net $\mathrm{N}$ input from crop residues in $\mathrm{N}$ budgets for subsequent crops-soil sequences.
\end{abstract}

Keywords: reduced tillage / crop residues / carbon / nitrogen / decomposition / modelling

\footnotetext{
*Auteur de correspondance : pascal.thiebeau@inra.fr
} 


\section{Introduction}

L'agriculture peut contribuer à la lutte contre le changement climatique, notamment par la fonction «puits» pour le carbone que représentent des sols agricoles, en favorisant des pratiques qui augmentent le taux de matières organiques des sols (MAAF, 2015). C'est notamment l'objectif du projet de recherche international « 4 pour 1000» (http:// 4 p1000.org/comprendre). L'une des façons d'y parvenir est de modifier les pratiques culturales permettant un accroissement des restitutions aux sols des résidus végétaux après leur récolte combiné à une réduction ou suppression du travail du sol (Arrouays et al., 2002; Brunnert, 1996; Seguin et Soussana, 2006), pratiques sur lesquelles repose l'agriculture de conservation (Scopel et al., 2013). La suppression du travail $\mathrm{du}$ sol présente d'autres atouts environnementaux. Elle favorise la biodiversité animale, la présence accrue de vers de terre ainsi que l'accumulation de matière organique dans la couche superficielle de sol favorisent respectivement l'infiltration et la rétention d'eau. Cela permet également de maintenir les sols en place : le paillis constitue une protection face à l'érosion éolienne comme hydrique et constitue un «effet tampon» face aux variations de températures (Waligora et al., 2010).

Dans les systèmes avec travail du sol réduit, qui mobilisent les techniques culturales simplifiées (TCS) et le semis direct (SD), les résidus de cultures sont maintenus à la surface du sol (SD), ou bénéficient d'un mélange partiel avec le sol dans les 5 à 7 premiers centimètres (TCS). La dynamique de décomposition des résidus, dans ces conditions, est beaucoup moins étudiée que dans des conditions de mélange au sol lors d'un labour profond. En général, les études existantes s'appuient sur des cinétiques de décomposition avec des sacs à litières (technique du litter-bag) ou la mise en place de microparcelles (Lynch et al., 2016; Pascault et al., 2010), qui s'éloignent des conditions réelles de décomposition sur des parcelles d'agriculteurs (taille des brins, contact avec le sol, accès de la macrofaune, etc.). La dynamique de décomposition a une incidence directe sur la libération des nutriments que ces résidus contiennent. Les enjeux de connaissance sont donc importants du point de vue opérationnel, puisque cette connaissance permettrait de mieux ajuster les apports d'éléments nutritifs aux cultures à venir, de l'intégrer dans les modèles de simulation d'émissions de gaz à effet de serre (GES) et les bilans environnementaux.

Dans ce contexte, l'objectif du travail présenté est d'étudier la dynamique de décomposition au sol de résidus de différentes cultures, dans des systèmes de production en agriculture de conservation, en conditions réelles de conduite d'exploitation, afin de mieux prédire leur décomposition et le devenir du carbone et de l'azote de ces résidus.

\section{Matériels et méthode}

Le travail a été réalisé sur trois exploitations de grandes cultures de la région «Grand Est» de la France, dans le contexte du projet de recherche PEPITES (Processus écologiques et processus d'innovations techniques et sociales en agriculture de conservation). Les exploitations étaient localisées sur les départements de l'Aube et de la Meuse, et pratiquaient les TCS et le SD depuis 5 à 10 ans, en 2009, lors du démarrage du projet. Pour chaque dynamique de décomposition, les mesures ont été réalisées depuis les récoltes 2009 et 2010, jusqu'à la fin de l'année 2011. Ces dynamiques ont été suivies au champ tant que la présence de résidus de la culture étudiée était clairement identifiable par rapport aux résidus de cultures suivantes.

\subsection{Dynamiques étudiées et méthode de prélèvement}

Nous avons suivi les dynamiques de décomposition des résidus de culture des espèces suivantes : maïs grain (Zea maïs L.), tournesol (Helianthus annuus L.), colza (Brassica napus L.), blé (Triticum aestivum L.) et escourgeon (Hordeum vulgare L.). Ces cultures présentent l'intérêt d'avoir des périodes de récolte décalées: entre le 16 juillet pour les premières céréales et le 28 octobre pour le maïs grain; de laisser sur le sol des quantités de résidus assez différentes (de $397 \mathrm{~g} \mathrm{MS.m}{ }^{-2}$ pour le tournesol à $1270 \mathrm{~g} \mathrm{MS.m}{ }^{-2}$ pour le maïs grain). Les suivis ont été réalisés sur 4 champs différents, tels que décrits sur la figure 1. Dans deux situations de TCS (maïs de l'automne 2009 et colza de l'été 2010), nous disposons de deux modalités de gestion des pailles :

- les résidus pré-enfouis dès la moisson, à l'aide d'un outil de travail superficiel du sol, avant la mise en place de la culture suivante (situations identifiées par l'extension « $\mathrm{CC} »)$;

- les résidus maintenus à la surface du sol jusqu'à la mise en place de la culture principale suivante (traitements identifiés par l'extension «Surf»).

Les échantillons de résidus ont été prélevés à intervalles de temps irréguliers, espacés d'un à plusieurs mois selon la saison. Le rythme des prélèvements avait pour objectif de minimiser l'impact des interventions en période de culture, sur la croissance et le rendement de la culture en croissance. Ces échantillons ont été prélevés à l'aide de cadres de $0,5 \times 0,5 \mathrm{~m}$ par la méthode du double transect telle que décrite par Thiébeau et Recous (2016). Brièvement, la méthode d'estimation repose :

- sur une série de prélèvements de résidus répartis sur une largeur de coupe de moissonneuse, afin d'intégrer un transect de répartition par le broyeur de la moissonneuse ;

- sur une série de prélèvements en diagonale par rapport à ce transect, afin de prendre en compte la variabilité de répartition des résidus créée en cours de progression de la moissonneuse.

Il était prélevé 1 cadre par mètre de largeur de coupe. Dans nos situations, le nombre de cadre prélevés a oscillé entre 10 et 14 lors de chaque estimation de biomasse, soit une surface totale de prélèvement variant de 2,5 à $3,5 \mathrm{~m}^{2}$. Les situations ayant subi le passage d'un outil de travail superficiel après la moisson ont fait l'objet d'une récolte sur l'ensemble de l'horizon de sol impacté par cette intervention, soit 4 à $7 \mathrm{~cm}$ de profondeur environ. Ces échantillons ont subi un premier tamisage sur le terrain, à l'aide d'un tamis de maille $0,5 \mathrm{~cm}$, afin de limiter le volume de l'échantillon à traiter au laboratoire ( $\mathrm{sol}+$ résidus), tout en respectant les parties de sol non travaillées. 


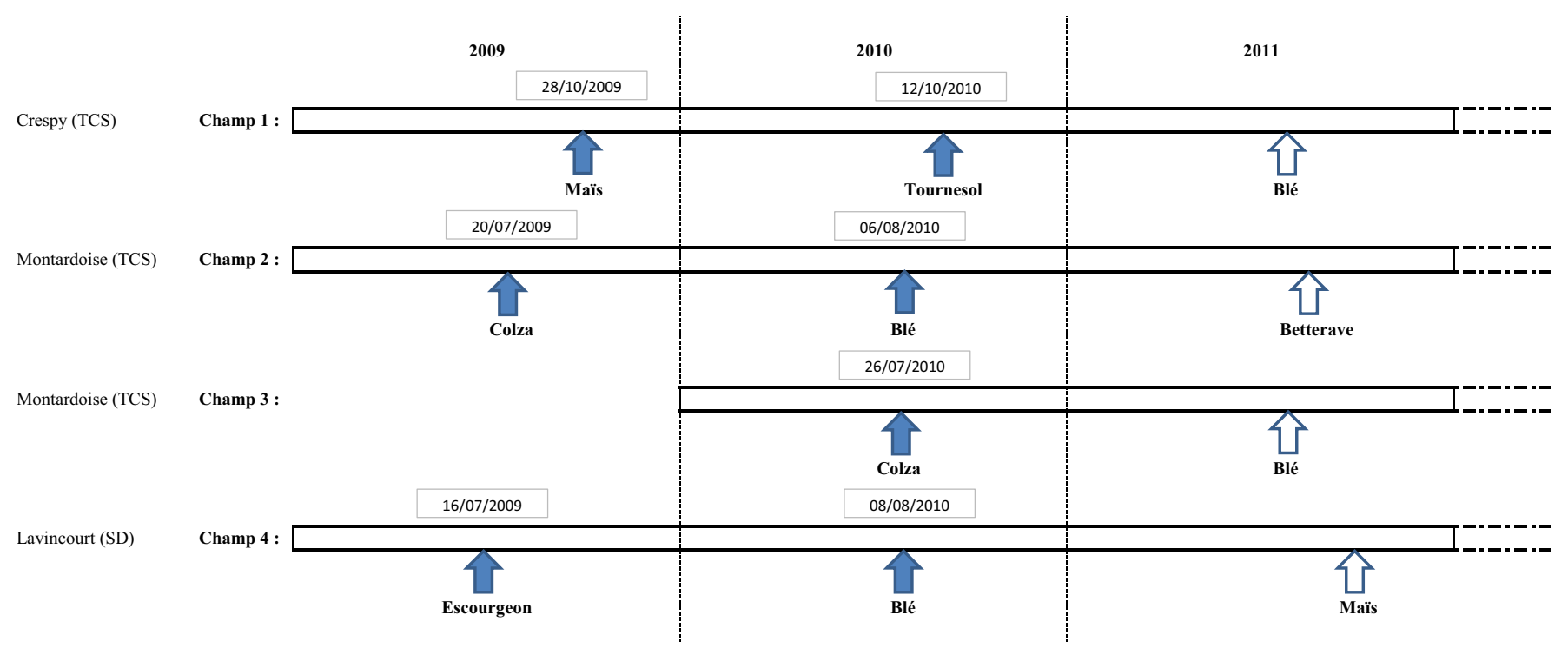

Fig. 1. Dispositif expérimental sur quatre exploitations agricoles, avec les successions de cultures. Les flèches pleines indiquent les résidus de cultures étudiés avec la date de moisson.

Fig. 1. Experimental design showing the crop rotation at four farm's location. Black arrows indicate the initial situations studied with the date of harvest.

Au laboratoire, seuls les échantillons ayant été en contact avec le sol ont fait l'objet d'un tamisage ( $2 \mathrm{~mm})$ à l'eau, afin de séparer les particules de sols des résidus végétaux. Les échantillons propres ont été passés en étuve ventilée pendant $48 \mathrm{~h}$ à $80^{\circ} \mathrm{C}$, tandis que les échantillons tamisés à l'eau l'étaient pendant $96 \mathrm{~h}$ à $80^{\circ} \mathrm{C}$. Chaque échantillon a été pesé dès sa sortie d'étuve pour la détermination de sa masse sèche.

\subsection{Analyses des teneurs en carbone et en azote total sur les échantillons secs}

Les échantillons secs ont été conservés en sachet papier avant leur broyage pour les analyses des teneurs en carbone (C) et en azote $(\mathrm{N})$ total. Après un broyage à $1 \mathrm{~mm}$ (broyeur à fléau), une fraction aliquote a été conservée pour un broyage à 80 microns (broyeur ultra-centrifuge à rotor), sur laquelle ont été effectuées les analyses de la teneur en $\mathrm{C}$ et $\mathrm{N}$ total, à l'aide d'un analyseur élémentaire NA2000 (Fison Instrument). Il détermine le carbone et l'azote total d'un échantillon par oxydation catalytique à haute température.

\subsection{Expression du temps en jours normalisés}

Compte tenu des dates de suivi des dynamiques de décomposition des résidus et des situations géographiques variées, et donc des conditions climatiques variables auxquelles les résidus sont exposés, il est important d'exprimer les observations sur la base d'une échelle de temps permettant de les comparer entre elles. Nous avons choisi d'utiliser la méthode des jours normalisés, largement utilisée dans plusieurs modèles de simulation de $\mathrm{C}$ et $\mathrm{N}$ (Brisson et al., 2009; Mary et al., 1999). Cette approche permet de transformer chaque jour calendaire en une durée exprimée par jour à une température et une humidité de référence, ou «jour normalisé » $(\mathrm{JN})$. La température de référence choisie ici est $15^{\circ} \mathrm{C}$. Cependant, nous avons négligé la fonction humidité de la méthode car il était impossible de connaître, de manière simple et précise, l'humidité du paillis à la surface du sol. Ce faisant, l'hypothèse implicite est que ce facteur est de second ordre par rapport à la température. L'équation utilisée est donc la suivante (Brisson et al., 2009):

$$
\mathrm{T}^{\circ} \cdot \mathrm{JN} 15=25 /\left(1+145 \times \exp \left(-0,120 \times \mathrm{T}^{\circ}\right)\right),
$$

si $\mathrm{T}^{\circ}<0$, alors $\mathrm{JN} 15=0$.

$\mathrm{T}^{\circ}$ est la température moyenne du jour exprimée en degré Celsius. La température moyenne de l'air collectée à $2 \mathrm{~m}$ sous abri (donnée météo France) a été utilisée. C'est une variable climatique très facilement accessible sur les sites de météo France et disponible gratuitement.

Pour nos situations, les dynamiques de décomposition des résidus de cultures sont donc présentées selon deux échelles: une échelle de temps correspondant aux dates de prélèvements, et une échelle de jours, exprimée en jours normalisés ( $\left.\mathrm{T}^{\circ} \cdot \mathrm{JN} 15\right)$ depuis la récolte.

\subsection{Traitements statistiques des données}

Toutes les données moyennes présentées sont accompagnées de leur erreur d'estimation standardisée, afin de pouvoir être comparées entre elles.

Les ajustements linéaires et non linéaires sont réalisés à l'aide du programme statistique de Sigma-Plot 12 (SPSS, Inc.) après validation du test de normalité (Shapiro-Wilk). Les critères d'évaluation des ajustements non linéaires retenus sont l'erreur quadratique au modèle (RMSE) et le coefficient de détermination $\left(R^{2}\right)$. Ils sont définis comme suit:

$$
R M S E=\sqrt{\frac{1}{n} \cdot \sum_{i=1}^{n}\left(P_{i}-O_{i}\right)^{2}} .
$$


$O$ et $P$ sont respectivement les valeurs observées et prédites, $n$ est le nombre de paires observées-simulées.

$R^{2}$ est le carré de la corrélation du coefficient de Person, entre les valeurs observées et les valeurs simulées. Il décrit la proportion de la variance totale des données observées qui peut être expliquée par le modèle. Il est calculé comme suit :

$$
R^{2}=\left[\frac{\sum_{i=1}^{n}\left(P_{i}-\bar{P}\right) \cdot\left(O_{i}-\bar{O}\right)}{\sigma_{p} \cdot \sigma_{o}}\right]^{2},
$$

avec $\bar{P}$ et $\bar{O}$ comme valeurs moyennes respectivement estimées et observées.

Le seuil de signification maximal retenu est $P<0,05$.

\section{Résultats}

$\mathrm{Au}$ cours de cette étude, les conditions de températures (Fig. 2A) ont été proches des normales saisonnières, hormis les mois de février 2010 et janvier 2011 significativement plus froids par rapport à la moyenne pluriannuelle. Par contre, les fortes pluviométries (de $83 \mathrm{~mm}$ versus $54 \mathrm{~mm}$ en moyenne pluriannuelle au mois de juillet 2009, et de $244 \mathrm{~mm}$ cumulés versus $105 \mathrm{~mm}$ en moyenne pluriannuelle en août et septembre 2010) ont provoqué des conditions humides de moisson et de récoltes des résidus.

Les dynamiques de matières sèches des résidus collectés au cours du temps sont présentées sur la figure $2 \mathrm{~B}$. Les situations initiales varient de 400 à plus de $1200 \mathrm{~g} \mathrm{MS} \mathrm{m}^{-2}$. Après une année, les masses restantes au sol sont inférieures à $200 \mathrm{~g}$ MS $\mathrm{m}^{-2}$ dans toutes les situations. En 2009, il n'y a pas d'effet du type de culture (Colza-Surf vs. Esc-Surf) sur la cinétique de pertes de MS, ni initialement, ni ultérieurement. Cette observation s'applique aussi à la cinétique de décomposition des résidus de maïs à partir de l'automne 2009, soit laissés à la surface du sol, soit superficiellement enfouis par le passage d'un outil (Maïs-Surf vs. Maïs-CC). En 2010, seul le colza pré-enfoui à l'aide d'un outil de travail superficiel (Colza-CC) se distingue significativement des autres situations, par une décroissance très importante de la MS en 2 mois.

Exprimer la dynamique de décomposition des résidus de culture en fonction de l'échelle de temps normalisé permet de comparer les cinétiques indépendamment des variations de température rencontrées. Le suivi expérimental au champ s'est déroulé sur 2,25 années ( 810 jours calendaires), soit du 16 juillet 2009 (premier prélèvement de résidus) au 4 octobre 2011 (date du dernier prélèvement). La dynamique la plus longue a été suivie du 20 juillet 2009 au 27 septembre 2011, soit 799 jours calendaires correspondant à 725 jours normalisés à $15^{\circ} \mathrm{C}$. Nous constatons (Fig. $3 \mathrm{~A}-\mathrm{C}$ ) que l'ensemble des observations obtenues pour les différentes cultures, les différentes modalités de gestion et les deux années de récolte forme un nuage de points décroissant au cours du temps, pour la quantité de matière sèche (MS) présente à la surface du sol (Fig. 3A), la quantité de carbone (QC) restant (Fig. 3B) ou la quantité d'azote (QN) restant (Fig. 3C). Les données collectées s'ajustent bien à l'aide d'une équation non linéaire avec un seul compartiment (MS ou $\mathrm{C}$ ou $\mathrm{N}$ ).
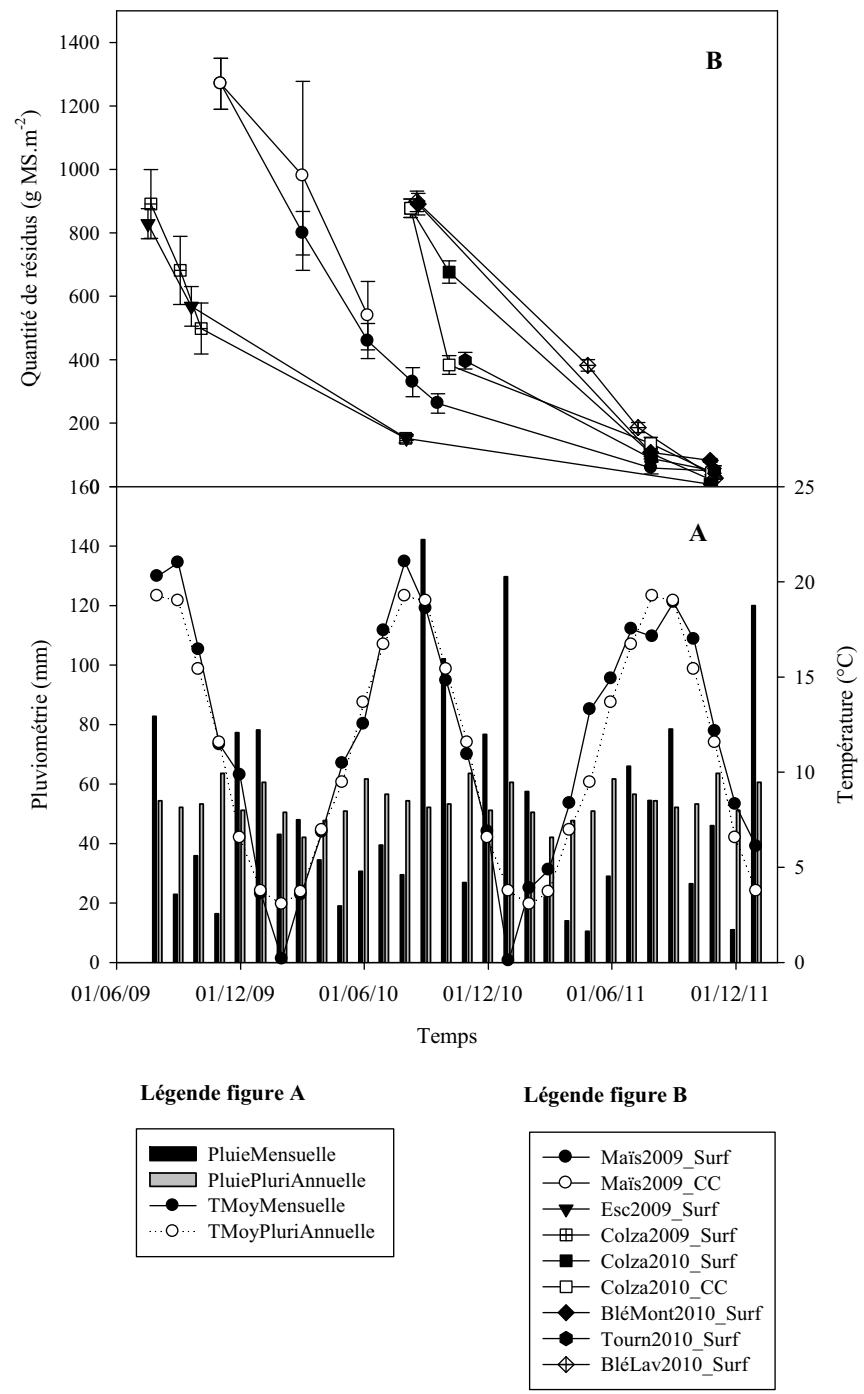

Fig. 2. A. Pluviométries et températures moyennes de l'air au cours de l'étude en comparaison aux données pluriannuelles respectives. B. Dynamiques de décomposition des résidus de cultures à la surface du sol au cours du temps pour les différentes situations.

Fig. 2. A. Rainfall and mean air temperatures during the study, and comparison with the mean pluriannual data on the same sites. B. Dynamics of crop residues decomposition over time for the various situations studied (bars are standard errors on replicates).

L'ajustement qui nous permet d'obtenir les meilleurs résultats statistiques est de la forme:

$$
\mathrm{Y}=\mathrm{a} \times\left[\operatorname{Exp}\left(-\mathrm{b} \times \sum \mathrm{JN} 15\right)\right]
$$

avec,

MS : $a=933,1$ et $b=0,006$ (RMSE $=145,0 \mathrm{~g}$ MS.m ${ }^{2}$; $\left.R^{2}=0,8552, P<0,001\right)$;

$\mathrm{QC}: \mathrm{a}=418,8$ et $\mathrm{b}=0,007 \quad\left(\mathrm{RMSE}=62,5 \mathrm{~g} \mathrm{C} \cdot \mathrm{m}^{2}\right.$, $\left.R^{2}=0,8675, P<0,001\right)$;

$\mathrm{QN}: \mathrm{a}=5,2 \mathrm{et} \mathrm{b}=0,005\left(\mathrm{RMSE}=1,1 \mathrm{~g} \mathrm{~N} . \mathrm{m}^{2}, R^{2}=0,7301\right.$, $P<0,001)$. 

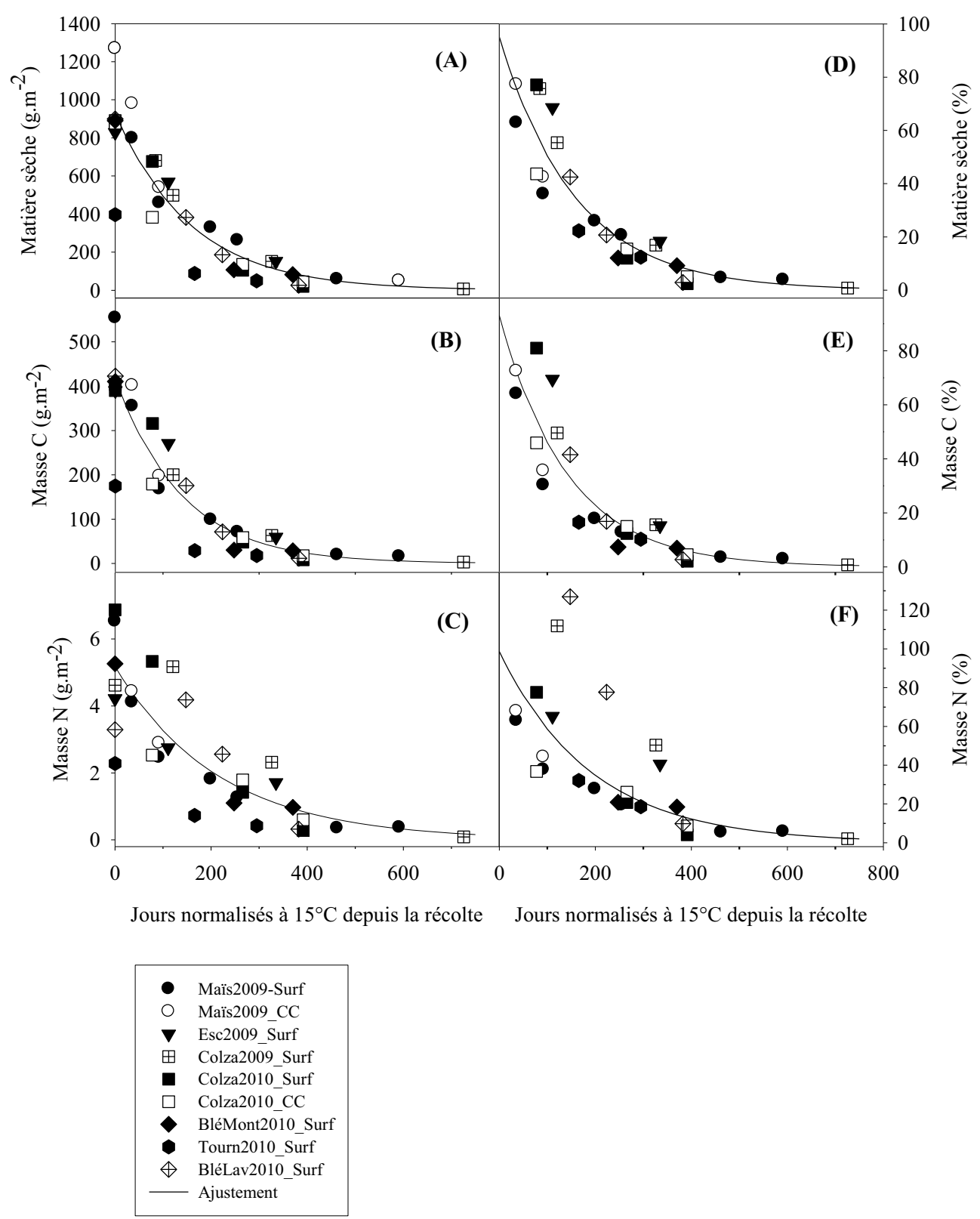

Fig. 3. Dynamiques de perte de matière sèche $(A)$, de carbone $(B)$ et d'azote $(C)$ de résidus de cultures, exprimées en masse $\left(\mathrm{g} . \mathrm{m}^{-2}\right)$ et exprimées en pourcentage de la quantité initiale $(\%)(\mathrm{D}-\mathrm{F})$ au cours du temps. Le temps est exprimé en somme de jours normalisés à $15^{\circ} \mathrm{C}$.

Fig. 3. Dynamics of crop residue dry matter loss (A), carbon (B) and nitrogen (C) expressed in g. $^{-2}$ and in percentage of initial mass, over time. Time is expressed as the sum of normalized days at $15^{\circ} \mathrm{C}$. Data are the mean of 10 to 14 replicates, depending of the harvest crop machine.

En exprimant ces dynamiques en fonction des quantités initiales de MS (Fig. 3D), C (Fig. 3E) et N (Fig. 3F), les paramètres de l'équation (1) deviennent les suivants :

MS : $\mathrm{a}=95,1$ et $\mathrm{b}=0,006\left(\mathrm{RMSE}=9,5 \% ; R^{2}=0,8541\right.$, $P<0,001)$;

$\mathrm{QC}: \mathrm{a}=93,1$ et $\mathrm{b}=0,007\left(\mathrm{RMSE}=9,9 \% ; R^{2}=0,8275\right.$, $P<0,001)$.

En ce qui concerne l'azote, l'ajustement à partir de l'équation (2) se révèle impossible. Nous constatons que la part augmente au-delà de $100 \%$ pour certaines situations, ce qui indique un enrichissement temporaire des résidus en $\mathrm{N}$ par rapport à la quantité initiale. Cela concerne les résidus de blé de 2010 et de colza de 2009. Pour parvenir à ajuster ces données, nous les avons linéarisées en passant par les logarithmes népériens. La fonction permettant leur ajustement est de la forme:

$$
\mathrm{Y}=\operatorname{Exp}\left[\left(\mathrm{a} \times \sum \mathrm{JN} 15\right)+\mathrm{b}\right]
$$

L'ajustement réalisé propose les valeurs suivantes des paramètres :

$\mathrm{QN}: \mathrm{a}=-0,005$ et $\mathrm{b}=4,592\left(\mathrm{RMSE}=0,525 ; R^{2}=0,7540\right.$, $P<0,001)$.

Cet ajustement, présentant un coefficient de détermination moins bon, reste de très bonne qualité statistique. 


\section{Discussion}

Le résultat original de ce travail est que l'ensemble des cinétiques de décomposition des résidus de cultures s'ajustent à l'aide d'un seul et même modèle, décrivant un seul « compartiment» de résidus sur cette échelle de temps de deux années environ. Ceci suggère qu'à l'échelle de temps considérée au champ (deux années), la composition biochimique des résidus n'a pas eu d'effet important sur le devenir du carbone, dans la gamme des espèces explorées ici. Pourtant, nous avons travaillé avec des quantités variables de résidus de plantes, ayant des caractéristiques différentes, et sur des exploitations et situations climatiques différentes au moment des récoltes et au cours des cinétiques de décomposition. Iqbal et al. (2013) ont montré sur les tiges de ces mêmes espèces une différence importante de la composition chimique (par exemple, le rapport fraction soluble : fraction lignine égale à 1,24 pour le blé et 3,92 pour le maïs) et des propriétés physiques (par exemple, la densité des tiges de $0,35 \mathrm{~g} \mathrm{~cm}^{-3}$ pour le blé et $0,10 \mathrm{~g} \mathrm{~cm}^{-3}$ pour le maïs). Or, ces cinétiques se superposent selon un ajustement proportionnel à la biomasse initialement présente, selon des jours «normalisés » sur le seul critère de la température de l'air. Les jours normalisés permettent de comparer à la fois des situations expérimentales de régions très différentes, mais aussi de comparer des essais au champ avec des expérimentations conduites au laboratoire en conditions contrôlées. Dans cette étude, ce résultat signifie aussi que la quantité de résidus, la composition biochimique des résidus et l'humidité, pourtant reconnues comme facteurs déterminants de la décomposition des pailles (Recous et al., 2017), ne l'ont pas été ici. Le facteur déterminant des cinétiques de décomposition semble avoir été piloté principalement, sur cette échelle de temps, par la température de l'air dans les conditions expérimentales de cette étude. Dans le contexte de travail qui a été le nôtre, l'humidité n'a sans doute pas été un facteur limitant puisque les pluies ont été régulièrement présentes, mais le paillis constitue aussi une couverture du sol qui limite l'évaporation de l'eau du sol (Coppens et al., 2006). Cette conclusion ne serait pas valable dans des régions où il ne pleut pas régulièrement. C'est le cas par exemple sous climat tropical, où, au contraire, le régime des pluies pilote la décomposition alors même que la température varie peu ou pas (Vanlauwe et al., 1995). Dans la situation explorée des exploitations agricoles de la région Grand Est, ce résultat suggère que l'on peut prédire de façon assez précise la décomposition des résidus de culture en utilisant, par exemple, les données météorologiques moyennes relatives à la température de l'air. Ce résultat devrait être confirmé par d'autres expérimentations, afin de définir l'aire géographique dans laquelle cette simplification est acceptable.

La libération du carbone et de l'azote contenus dans ces résidus suit globalement la dynamique de la matière sèche, ce qui était prévisible en particulier pour le carbone, dont la teneur reste quasi constante dans les résidus. Le moins bon ajustement des données relatives à l'azote peut s'expliquer par les variations possibles de la teneur en azote des résidus, liées aux processus d'organisation microbienne de l'azote sur les résidus eux-mêmes par les décomposeurs (Recous et al., 2015). On observe notamment une rétention plus grande de l'azote, voire un accroissement net de la quantité d'azote, en début de décomposition, sur les cultures de colza en 2009 et blé en 2010, alors que la perte de matière sèche et de carbone est en cours. L'ajustement de très bonne qualité $(P<0,001)$, et unique pour plusieurs types de résidus de culture et plusieurs années, suggère qu'il est possible de prédire assez simplement la dynamique de l'azote des résidus, dans le bilan à la parcelle, pour en tenir compte par exemple dans le calcul de la fertilisation azotée.

Les deux cultures pour lesquelles nous disposons d'une comparaison «avec» ou «sans» pré-enfouissement des résidus dès la récolte montrent des comportements différents des dynamiques. Dans l'une des situations (maïs 2009), le préenfouissement ralenti légèrement la perte de matière sèche au cours des deux mois qui suivent l'opération culturale, sans présenter de différence significative avec les résidus laissés à la surface du sol, pour suivre, ensuite, la même dynamique. Dans l'autre situation (colza 2010), le pré-enfouissement montre une perte significativement plus importante de matière sèche au cours des deux mois qui suivent la pré-incorporation au sol, mais les différences disparaissent ensuite. Ces situations impactent peu, en définitive, la dynamique globale de décomposition des résidus au champ. Le pré-enfouissement des résidus dès la récolte, qui favorise le contact entre le sol et les résidus, ne présente donc pas d'intérêt notable sur leur dynamique de décomposition dans les situations culturales explorées par cette étude. On pourrait attendre, au vu des résultats de la littérature, que les résidus enfouis soient décomposés plus rapidement que ceux à la surface du sol, en raison d'une humidité plus favorable et d'une accessibilité plus importante des décomposeurs à l'azote minéral indispensables à leur croissance (Coppens et al., 2006). Le pré-enfouissement a cependant d'autres atouts agronomiques qui pourraient le justifier: levée plus rapide des graines de la culture récoltée afin d'éviter une levée indésirable dans la culture suivante, mais aussi levée des adventices en lutte préventive, installation plus rapide de cultures «piège à nitrates» semées dès la moisson (Colbach et Vacher, 2014). Cette technique permet également de réaliser une lutte contre les campagnols et autres prédateurs des cultures lorsque que leur infestation est jugée conséquente par l'exploitant (Delattre et Giraudoux, 2009).

\section{Conclusion}

Ce travail a permis de montrer l'effet prépondérant de la température sur la décomposition de résidus de culture laissés soit en paillis à la surface du sol, soit légèrement incorporés par un travail du sol superficiel, dans des parcelles d'exploitations menées en travail du sol simplifié ou sans labour dans la région Grand Est de la France. Par l'utilisation d'ajustements simples basés sur une échelle de temps normalisé, il est possible d'estimer les dynamiques de pertes de matière sèche, de carbone et d'azote de ces résidus, et donc pour ce dernier élément, indirectement, de fourniture et d'immobilisation d'azote par les résidus de culture au sol. Ces résultats peuvent donc être inclus de manière simple, à partir de la connaissance des quantités restituées et des températures moyennes de l'air, dans des outils d'aide à la décision pour la gestion des matières organiques et des éléments fertilisants dans les systèmes agricoles pratiquant le travail réduit du sol.

Remerciements. Les auteurs remercient Messieurs Ferté, Gubelin et Lemey, exploitants agricoles, qui ont accepté de 
mettre à notre disposition un peu de leur temps et leurs champs afin que nous puissions conduire cette étude. Nous remercions l'Agence nationale de la recherche (ANR) pour le soutien financier au programme Systerra, projet PEPITES (Processus écologiques et processus d'innovations techniques et sociales en agriculture de conservation), financé de 2009 à 2013.

\section{Références}

Arrouays D, Balesdents J, Germon J-C, Jayet P-A, Soussana J-F. 2002. Contribution à la lutte contre l'effet de serre. Stocker du carbone dans les sols agricoles en France? Expertise scientifique collective, Synthèse, Inra, $32 \mathrm{p}$.

Brisson N, Launay M, Mary B, Beaudoin N. 2009. Conceptual basis, formalizations and parameterization of the STICS crop model. Versailles: Quae, 297 p.

Brunnert H. 1996. The significance of agriculture and forestry for the global carbon cycle. How can they contribute the sequester increasing amounts of $\mathrm{CO}_{2}$ in the atmosphere. Berichte Uber Landwirtschaft 74(1): 44-65.

Colbach N, Vacher C. 2014. Travail du sol et gestion de la flore adventice. In: Labreuche J, Laurent F, Roger-Estrade J, eds. Faut-il travailler le sol? Acquis et innovations pour une agriculture durable. Versailles: Quae, pp. 113-125.

Coppens F, Garnier P, De Gryze S, Merckx R, Recous S. 2006. Soil moisture, carbon and nitrogen dynamics following incorporation and surface application of labelled crop residues in soil columns. European Journal of Soil Science 57: 894-905.

Delattre P, Giraudoux P. 2009. Le campagnol terrestre : prévention et contrôle des populations. Versailles: Quae, $304 \mathrm{p}$.

Iqbal A, Beaugrand J, Garnier P, Recous S. 2013. Tissue density determines the water storage characteristics of crop residues. Plant and Soil 367: 285-299.

Lynch MJ, Mulvaney MJ, Hodges SC, Thompson TL, Thomason WE. 2016. Decomposition, nitrogen and carbon mineralization from food and cover crop residues in the central plateau of Haiti. SpringerPlus 20165: 973.

MAAF. 2015. Contribution de l'agriculture à la lutte contre le changement climatique : Stéphane LE FOLL annonce le lancement d'un projet de recherche international: le «4 pour $1000 »$. Communiqué de presse du 17 mars. http://agriculture.gouv.fr/ contribution-de-lagriculture-la-lutte-contre-le-changement-clima tique-lancement-dun-projet-de.

Mary B, Beaudoin N, Justes E, Machet JM. 1999. Accumulation of nitrogen mineralization and leaching in fallow soil using a simple model. European Journal of Soil Science 50(4): 549-566.

Pascault N, Nicolardot B, Bastian F, Thiébeau P, Ranjard L, Maron PA. 2010. In situ dynamics and spatial heterogeneity of soil bacterial communities under different crop residue management. Environmental Microbiology 60: 291-303.

Recous S, Chabbi A, Vertès F, Thiébeau P, Chenu C. 2015. Fertilité des sols et minéralisation de l'azote: sous l'influence des pratiques culturales, quels processus et interactions sont impliqués? Fourrages 223: 189-196.

Recous S, Lashermes G, Bertrand I. 2017. Couplages et contrôles des cycles du carbone et de l'azote par les communautés microbiennes dans les sols cultivés. In: Briat JF, Job D, eds. Les sols et la vie souterraine: des enjeux majeurs en agroécologie. Versailles: Quae.

Scopel E, Triomphe B, Affholder F, Macena Da Silva FA, Corbeels M, Valadares JH, et al. 2013. Conservation agriculture cropping systems in temperate and tropical conditions, performances and impacts. A review. Agronomy for Sustainable Development 33(1): 113-130.

Seguin B, Soussana JF. 2006. Le réchauffement climatique (prédictions futures et observations récentes) en lien avec les émissions de GES. Fourrages 186: 139-154.

Systat Software. 2010. SigmaPolt 12. https://systatsoftware.com/ products/sigmaplot (dernière consultation le 11/07/2017).

Thiébeau P, Recous S. 2016. Une méthode pour quantifier les biomasses de résidus de récolte à la surface des sols après la moisson. Cahiers Agricultures 25(4): 45001.

Vanlauwe B, Vanlangenhove G, Merckx R, Vlassak K. 1995. Impact of rainfall regime on the decomposition of leaf litter with contrasting quality under subhumid tropical conditions. Biology and Fertility of Soils 20: 8-16.

Waligora C, Thomas F, Recous S, Thiébeau P, Iqbal A. 2010. Le mulch, protection et garde-manger à la fois. Techniques Culturales Simplifiées 60: 18-26.

Citation de l'article : Thiebeau P, Recous S. 2017. Dynamiques de décomposition des résidus de cultures sur des exploitations pratiquant l'agriculture de conservation en région Grand Est, France. Cah. Agric. 26: 65001. 